REVIEW

\title{
Neurodegenerative disorders: Parkinson's disease and Huntington's disease
}

\author{
S M Hague, S Klaffke, O Bandmann
}

J Neurol Neurosurg Psychiatry 2005;76:1058-1063. doi: 10.1136/jnnp.2004.060186

Parkinson's disease and Huntington's disease are both model diseases. Parkinson's disease is the most common of several akinetic-rigid syndromes and Huntington's disease is only one of an ever growing number of trinucleotide repeat disorders. Molecular genetic studies and subsequent molecular biological studies have provided fascinating new insights into the pathogenesis of both disorders and there is now real hope for disease modifying treatment in the not too distant future for patients with Parkinson's disease or Huntington's disease.

See end of article for authors' affiliations

Correspondence to Dr Oliver Bandmann, Academic Neurology Unit, E Floor Medical School, Beech Hill Road, Sheffield S10 2RX, UK; o.bandmann@ sheffield.ac.uk

Received 3December 2004 In revised form 29 April Accepted 11 May 2005

\section{PARKINSON'S DISEASE}

Parkinson's disease is the second most common neurodegenerative disorder after Alzheimer's disease. The age adjusted prevalence for Parkinson's disease and other types of parkinsonism in the United Kingdom is 254/100 000, but the prevalence rises with age from $0.143 \%$ in the 50 to 59 year old population to $1.75 \%$ in the population aged 80 years or older. ${ }^{1}$ The pathological hallmarks are dopaminergic cell loss in the substantia nigra and the presence of Lewy bodies and Lewy neurites. Lewy bodies and dystrophic Lewy neurites are cytoplasmic accumulations of aggregated proteins.

Research into the pathogenesis of this disorder in the 1980s and early 1990s predominantly focused on oxidative stress and impaired function of the mitochondrial respiratory chain. Since the mid-1990s, scientific progress has been mainly the result of molecular genetic research and further studies investigating the physiological role of the mutated genes/proteins, the functional consequences of the disease causing mutations, and a subsequent investigation of the affected pathways in sporadic Parkinson's disease (table 1).

$\alpha$-Synuclein (A-S) was the first identified Parkinson's disease gene (PARKl). To date, only three autosomal dominantly inherited point mutations (Ala53Thr, Ala30Pro, and Glu46Lys) have been described. ${ }^{2-4}$ The Ala53Thr mutation has been detected in several families of Mediterranean origin with autosomal dominantly inherited Parkinson's disease, but the Ala30Pro mutation has been discovered only in one small German family. ${ }^{2}{ }^{3}$ Within the families with either the Ala53Thr or the Ala30Pro mutation, the ratio of normal (wild type) to mutant A-S correlates with the disease severity: the more severely patients are affected, the less mutant A-S is expressed (because PARKl is autosomal dominantly inherited, both a normal and a mutant copy of the gene (allele) are present). Thus the ratio between normal and mutant A-S (haploinsufficiency) may be important for disease progression and severity. ${ }^{5}$ The average age of onset in the famous Contursi kindred (in which the Ala53Thr mutation was first identified) was 45.6 years. Affected family members presented with typical parkinsonian features such as resting tremor, bradykinesia, and gait disturbance, but progressed more rapidly than typical Parkinson patients. ${ }^{6}$ Subsequently, additional features such as central hypoventilation, orthostatic hypotension, prominent myoclonus, and urinary incontinence have been described in a different Ala53Thr family, indicating a wide range of phenotypes for Ala53Thr mutation carriers. ${ }^{7}$ Cognitive impairment is a frequent and early symptom in Ala30Pro mutation carriers, but the phenotype is otherwise similar to sporadic Parkinson's disease, with an age of onset ranging from 54 to 76 years. $^{8}$ The most recently discovered Glu46Lys mutation not only causes parkinsonism but also results in clinical and pathological features characteristic of Lewy body dementia. ${ }^{4}$ It has been suggested that single A-S polymorphisms or haplotypes formed by a combination of several A-S sequence variants may be a risk factor for isolated Parkinson's disease, but this has not been consistently confirmed by others. ${ }^{9-11}$ A-S, but not the closely related $\beta$ and $\gamma$ synucleins, is consistently found in Lewy bodies, not only in those rare families with a genomic A-S mutation but in all cases of Lewy-body Parkinson's disease. ${ }^{12} \mathrm{~A}-\mathrm{S}$ is also present in the glial cytoplasmic inclusion bodies typical for multiple system atrophy. ${ }^{13}$

The physiological role of A-S is still largely unknown, but its localisation at presynaptic terminals and some functional studies indicate a possible role in synaptic plasticity and vesicular transport. ${ }^{12}$ A-S knockout mice have a defect in dopamine release and reuptake, supporting a role for A-S in the regulation of dopamine transmission. ${ }^{14}$ How does A-S contribute to the cell death observed in Parkinson's disease and how does it fit in with previously recognised pathogenic mechanisms such as oxidative stress? Wild type A-S expression confers an increased resistance to various apoptotic insults, whereas mutant A-S

Abbreviations: A-S, $\alpha$-synuclein; COX2, cyclooxygenase 2; CBP, CREB binding protein; MPTP, 1methyl-4-phenyl-1,2,3,6 tetrahydropyridine 
Table 1 Familial forms of Parkinson's disease: genes, chromosomal loci, and mode of inheritance

\begin{tabular}{|c|c|c|c|}
\hline Locus/gene & $\begin{array}{l}\text { Chromosomal } \\
\text { location }\end{array}$ & $\begin{array}{l}\text { Inheritance } \\
\text { pattern }\end{array}$ & Clinical features \\
\hline PARK-1/ $\alpha$-synuclein & $4 q 21-q 23$ & $A D$ & Late onset \\
\hline PARK-2/parkin & $6 q 25.2-q 27$ & $A R$ & Juvenile onset, slow progression, focal dystonia \\
\hline PARK-3 & $2 \mathrm{p} 13$ & $A D$ & Late onset \\
\hline $\begin{array}{l}\text { Formerly PARK- } 4 / \alpha- \\
\text { synuclein triplication }\end{array}$ & $4 q 21-q 23$ & $A D$ & $\begin{array}{l}\text { Early onset, rapid progression, postural tremor, late } \\
\text { dementia }\end{array}$ \\
\hline PARK-5//UCHL-1 & $4 \mathrm{p} 14$ & $A D$ & Late onset \\
\hline PARK-6/PINK-1 & 1 p35-p36 & $A R$ & Early onset, slow progression \\
\hline PARK-7/DJ-1 & $1 \mathrm{p} 36$ & $A R$ & Early onset, slow progression \\
\hline PARK-8/LRRK-2 & $12 \mathrm{p} 11.2-\mathrm{q} 13.1$ & $A D$ & Late onset \\
\hline PARK-10/unknown & $1 \mathrm{p} 32$ & & Late onset \\
\hline PARK-11/unknown & $2 q 36-q 37$ & & Late onset \\
\hline NA/synphilin-1 & $5 q 23.1-q 23.3$ & $A D$ & Late onset \\
\hline NA/NR4A2 & $2 q 22-q 23$ & $A D$ & Late onset \\
\hline
\end{tabular}

$A D$, autosomal dominant; $A R$, autosomal recessive.

results in increased apoptotic response and enhanced susceptibility to oxidative stress. ${ }^{12}$ More importantly, different mechanisms lead to the aggregation of A-S with subsequent formation of protofibrils, fibrils, and eventually conversion to Lewy bodies. ${ }^{15}$ Wild type A-S is natively unfolded but forms fibrils at increasing concentrations, and its overexpression in marmosets results in dopaminergic cell loss as well as A-S positive cytoplasmic inclusions. ${ }^{12}{ }^{16}$ Fibril formation is accelerated by the A53T mutation of A-S, but also by oxidative stress, heavy metals, pesticides, and l-methyl-4-phenyl-1,2,3,6 tetrahydropyridine (MPTP). ${ }^{12}{ }^{15}$ Fascinatingly, A-S knockout mice show resistance to MPTP but increased susceptibility to a different neurotoxin, rotenone. ${ }^{17}$ Aggregation is also facilitated by nitration, indicating the relevance of post-translational modification processes. ${ }^{18}$ Why does A-S cause selective neurodegeneration in Parkinson's disease? This may at least partly reflect the fact that A-S renders endogenous levels of dopamine toxic in cultured human dopaminergic neurones, this toxicity being mediated by reactive oxygen species. In contrast, A-S is not toxic in non-dopaminergic human cortical neurones, but rather shows neuroprotective activity. ${ }^{19}$ The recent discovery of A-S triplication as a further cause for Parkinson's disease indicates that not only mutated but also non-physiological amounts of A-S can lead to nigral cell death. ${ }^{20}$

Parkin is the second Parkinson's disease gene (PARK2). Parkin mutations are typically detected in autosomal recessively inherited young onset parkinsonism with dystonia, sleep benefit, and the tendency to develop L-dopa induced dyskinesias, ${ }^{21}$ but some patients with Parkin mutations have a later age of onset (up to 65 years) and are clinically indistinguishable from patients with idiopathic Parkinson's disease. ${ }^{21} 22$ Disease progression is considerably slower in patients with parkin mutations than in idiopathic, Lewy body Parkinson's disease. ${ }^{23}$ Parkin localises to the Lewy bodies of Parkinson's disease in those patients who do not carry Parkin mutations. In contrast, Lewy bodies are absent in patients with homozygous Parkin mutations. Unusual presentations such as levodopa unresponsive parkinsonism combined with cerebellar and pyramidal tract dysfunction have also been reported. ${ }^{24}$ Interestingly, heterozygote mutations can also be found in some patients with young onset parkinsonism, and some asymptomatic heterozygote mutation carriers also show significant striatal dopaminergic dysfunction on ${ }^{18} \mathrm{~F}$-dopa positron emission tomography (PET).$^{23}$ A polymorphism $(-258 \mathrm{~T} / \mathrm{G})$ in the promoter region of the parkin gene may be associated with idiopathic Parkinson's disease, but this finding awaits confirmation by others. ${ }^{25}$
A mutation in the gene encoding ubiquitin carboxyterminal hydrolase 1 (UCHLl) has been described in a single small German kindred with autosomal dominantly inherited Parkinson's disease (PARK5) and the S18Y polymorphism in the UCHLl gene may be associated with decreased risk for Parkinson's disease..$^{26} 27$

Both parkin and UCHLl are involved in the ubiquitin dependent degradation of intracellular misfolded, unassembled, or damaged proteins by the proteasome, a multicatalytic complex. Parkin is an E3 ligase responsible for the attachment of ubiquitin to substrates, whereas UCHL-1 removes polyubiquitin chains once the substrate has been attached to the proteasome. ${ }^{28}$ Glycosylated A-S and the A-S interacting protein synphilin-1 are among the recognised substrates of parkin (fig 1). Mutations in either the parkin gene or UCHLl lead to impaired protein degradation. ${ }^{29}$ Expression of A53T mutant A-S also induces disruption of the ubiquitin dependent proteolytic system in cell culture models. ${ }^{30}$ Subsequent work showed reduced enzymatic activity and impaired structural integrity of the proteasome in the substantia nigra of patients with sporadic Parkinson's disease. ${ }^{31}$ These findings suggest that failure of the ubiquitin-proteasome system to achieve adequate clearance of unwanted proteins may underlie vulnerability and degeneration of the substantia nigra, even in sporadic Parkinson's disease. The expression of proteasome subunits in the mesencephalon of rats declines with age. One could therefore speculate that the age dependent prevalence of Parkinson's disease reflects a lifetime accumulation of abnormal intracellular proteins (for example, from oxidative damage) and an increasingly incompetent ubiquitin-proteasomal complex. However, proteasome subunits are also selectively vulnerable to oxidative stress, and the structural and functional integrity of the proteasome depends on sufficient ATP production. Thus the observed impairment of the proteasome in Parkinson's disease may merely be secondary, because of oxidative stress and impaired complex I activity of the mitochondrial respiratory chain (see McNaught $e t a^{31}$ for more detailed discussion).

Autosomal recessive mutations in the DJ-1 gene (PARK7) were originally identified in two consanguineous families from genetically isolated communities in the Netherlands and Italy, but subsequently also in other populations. ${ }^{32}$ Behavioural and psychiatric disturbances at onset or early in the disease course, and focal dystonia including blepharospasm, were noticed in both families. ${ }^{33}$ DJ-1 mutations lead to reduced DJ-1 protein stability, and the mutant protein is rapidly degraded through the ubiquitin-proteasome system. ${ }^{34}$ Knockdown of DJ-1 in cell culture systems leads to increased 


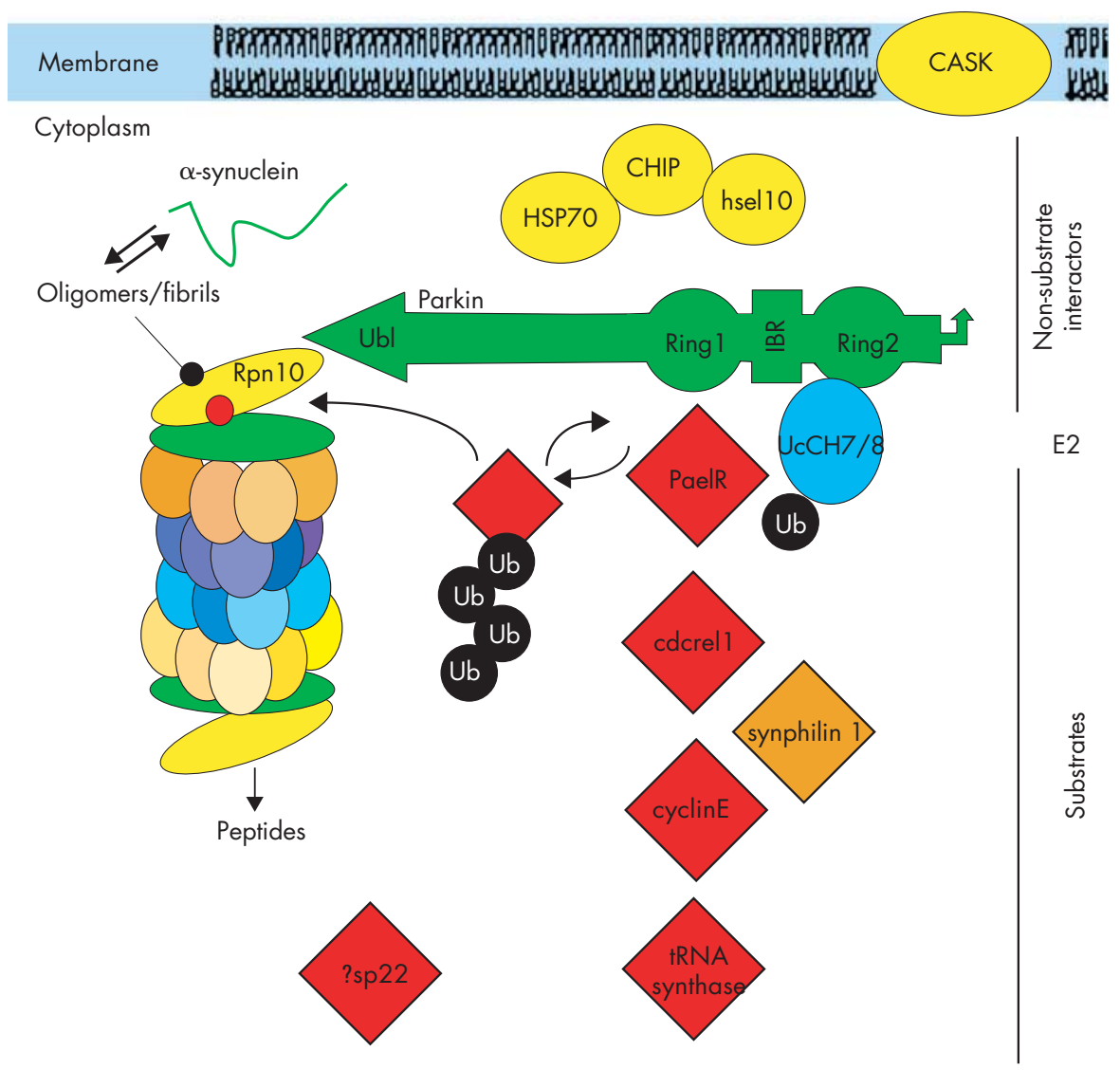

Figure 1 The ubiquitin-proteasome system. Parkin, shown in green, consists of several distinct domains, a ubiquitin-like domain (Ubl) and two RING fingers at the C-terminus, separated by an in between ring (IBR) domain. Parkin has been shown to bind to several substrates (shown in red), which are oriented with the PARKIN domains to which they bind. The RING-IBR-RING domain also binds various non-substrate interactors (yellow), namely the heat shock protein Hsp70, the C-terminal HSP interacting protein CHIP, and hsel10. Additional non-substrate interactors bind to the C-terminus of Parkin, including, CASK/lin10, and a protein in the cap of the proteasome, Rpn10. The $\mathrm{N}$ terminus of Parkin is responsible for binding to the proteasome. RING2 recruits E2 enzymes (blue), which themselves carry ubiquitin. Several rounds of ubiquitin addition build a polyubiquitin chain on the substrate, which is subsequently degraded by the proteasome. Mutant and wild type $\alpha$-synuclein (to a lesser degree) inhibit the proteasome. (Reproduced with kind permission of Mark Cookson and Sage Publications, Beverly Hills, California, USA).

susceptibility to oxidative stress and proteasome inhibition. ${ }^{35}$ DJ-1 co-localises with tau inclusions, but Lewy bodies are DJ-1 negative and necropsy reports on patients with a DJ-1 mutation are not yet available. ${ }^{36} 37$

Autosomal recessively inherited mutations in the PTEN induced kinase 1 on chromosome 1 p36 (PARK6) were also initially discovered in Parkinson patients from consanguineous families, but subsequently reported in sporadic patients with early onset Parkinson's disease as well. ${ }^{38} 39$ PINKl is localised to the mitochondria. Wild type, but not mutant, PINKl protein protects against stress induced mitochondrial dysfunction and apoptosis. ${ }^{38}$ DJ-1 polymorphisms, but not PINKl polymorphisms (naturally occurring sequence variants), may confer increased susceptibility to sporadic Parkinson's disease. ${ }^{40} 41$

Most recently, leucine-rich repeat kinase 2 (LRRK2) or dardarin was identified as the causative gene in families linked to the autosomal dominantly inherited PARK8 locus on chromosome 12pl1.2-q13.1. ${ }^{42}{ }^{43}$ The predicted product of the LRRK2 gene is a large protein with 2527 amino acids; sequence comparison suggests that it may function as a protein kinase. Necropsy diagnoses of six mutation carriers included abnormalities consistent with Lewy body Parkinson's disease, diffuse Lewy body Parkinson's disease, nigral degeneration without distinctive histopathology, progressive supranuclear palsy-like pathology, and clinical diagnoses of parkinsonism with dementia or amyotrophy, or both, with their associated pathology, were also noted. ${ }^{43}$ Thus LRRK2 may not only play a role in the pathogenesis of Parkinson's disease as such, but also of other neurodegenerative disorders. Subsequent studies have described the Gly2019Ser mutations in both familial and sporadic forms of Parkinson's disease in several distinct populations, with a frequency ranging from $1 \%$ to $6 \% .^{44-46}$

More information about the penetrance and other clinically relevant aspects of this mutation are needed, but genetic testing for the Gly2019Ser mutation may be the first diagnostic genetic test for Parkinson's disease to enter clinical practice.

The NR4A2 (also known as NURR1) gene encodes a member of the nuclear receptor superfamily and is essential for the differentiation of nigral dopaminergic neurones. Heterozygote mutations in NR4A2 have been detected in 10 of 107 patients with autosomal dominantly inherited Parkinson's disease, but not in sporadic disease or controls. Age of onset and clinical features were not different from typical Parkinson's disease. The mutations result in a marked decrease of NR4A2 mRNA levels and downregulate the transcription of the tyrosine hydroxylase gene. ${ }^{47}$ Numerous subsequent studies failed to detect any sequence variants in 
other Parkinson patient cohorts, and there is now at least the suspicion that the observed sequence changes of NR4A2 in the original study may simply reflect a haplotype cosegregating with the disease in that particular population rather than a disease causing mutation as such.

In addition to genetic factors, exposure to toxins is the only other universally recognised risk factor for Parkinson's disease. Systemic application of the herbicide paraquat has been shown to kill dopaminergic neurones in the substantia nigra in rodents. ${ }^{48}$ The pesticide rotenone, a specific inhibitor of mitochondrial complex I, is highly lipophilic, easily crosses biological membranes, and does not selectively accumulate in nigral neurones. Nevertheless, chronic systemic administration of rotenone has been reported to result in selective nigral degeneration. ${ }^{49}$ Others have, however, described a multisystem degeneration in rats treated with rotenone, indicating non-selective neurotoxicity. ${ }^{50}$ Interestingly, non-toxic concentrations of rotenone and the inflammogen lipopolysaccharide synergistically induced cell death in a dopaminergic mesencephalic cell culture model. ${ }^{51}$ Microglial generation of reactive oxygen species appeared to be a key contributor to this synergistic neurotoxicity. Other microglia originated factors such as nitric oxide, tumour necrosis factor $\alpha$, and interleukin $1 \beta$ may further contribute to the neurodegenerative process observed in Parkinson's disease. ${ }^{52}$ Inflammatory processes associated with an increased expression of cyclooxygenase 2 (COX2), the rate limiting enzyme in prostaglandin $E_{2}$ synthesis, and raised levels of prostaglandin $E_{2}$ have been implicated in the pathogenesis of several neurodegenerative disorders, and upregulation of COX2 has now also been observed in dopaminergic neurones of both Parkinson's disease and MPTP mice. Interestingly, COX-2 inhibition does not protect against MPTP induced dopaminergic neurodegeneration by mitigating inflammation. Rather, COX2 inhibition prevents the formation of the oxidant species dopaminequinone. COX-2 inhibitors can penetrate the blood-brain barrier and it was thus initially hoped that these drugs might be of future therapeutic use in Parkinson's disease. ${ }^{53}$ The recently reported side effect profile of COX-2 inhibitors, however, has obviously cast some doubt on this.

The discovery of the different gene defects described above highlighted the relevance of the ubiquitin-proteasome pathway for neuronal cell death in Parkinson's disease. The function of this pathway can also be influenced by naturally or synthetic proteasome inhibitors. Treatment of rats with proteasome inhibitors closely mimics Parkinson's disease in rodents. ${ }^{54}$ The treated rats develop progressive parkinsonism with dykinesia, rigidity, and tremor. Necropsy analysis showed striatal dopamine depletion, dopaminergic cell death with inflammation, and apoptosis in the substantia nigra pars compacta. Lewy bodies were additionally described in a subset of the remaining neurones. This model potentially offers a substantial improvement over the previously described model systems as it appears to resemble the cardinal features of Parkinson's disease more closely.

Currently, only symptomatic treatment is available for Parkinson's disease. The study of the underlying genetic and cellular defects in both familial and sporadic disease provides an opportunity to identify novel targets and tools to ameliorate disease progression and possibly even provide a cure. Overexpression of molecular chaperones such as HSP40 or HSP70 markedly reduces the formation of inclusion bodies. ${ }^{55}$ Administration of geldanamycin induces the expression of heat shock proteins and protects against A-S toxicity in Drosophila Parkinson's disease models. ${ }^{56}$ Thus the induction of such molecular chaperones may become an exciting new type of disease modifying treatment for Parkinson's disease.

Too much A-S may not only lead to Parkinson's disease in those comparatively rare families with duplication or triplication of the A-S gene, but also in those sporadic patients who produce too much A-S because of the presence of a particular sequence variant in the promoter region of A-S. ${ }^{57}$ A reduction of the A-S protein levels in the affected individuals may be a further promising therapeutic avenue. Indeed, a reduction in A-S protein levels has been achieved in a rodent model with overexpression of virus delivered parkin. $^{58}$ The common mutations of Gly2019 and Ile2020 in PARK8/LRRK2 may alter the kinase activity of this protein. The modification of kinase activity with specific inhibitors provides a very attractive and achievable treatment strategy, which could become useful in the treatment of both familial and sporadic disease.

\section{HUNTINGTON'S DISEASE}

Polyglutamine diseases such as Huntington's disease, Kennedy's disease, dentatorubro-pallidoluysian atrophy (DRPLA), and some of the autosomal dominantly inherited spinocerebellar ataxias result from an increased number of CAG nucleotide repeats that encode polyglutamine tracts within the corresponding gene products. The various proteins show no sequence homology outside the polyglutamine tract, span different lengths, have different cellular localisations and, where known, different functions. ${ }^{59}$ A relatively modest quantitative change of approx $10-20 \%$ in repeat length differentiates between normal (in Huntington's disease up to 35 repeats) and progressive neurodegeneration (in Huntington's disease 40 repeats or more). Subjects with 36-39 repeats have reduced penetrance for Huntington's disease. Longer expansions correlate with earlier onset and more severe disease. Rare cases of Huntington patients homozygous for an expansion (pathological expansion on both alleles) develop a more severe phenotype, but the presence of two expanded alleles rather than one does not seem to influence the age of onset. ${ }^{60}$

Proteins with elongated polyglutamine tracts misfold and aggregate as antiparallel $\beta$ strands termed "polar zippers" and form intranuclear inclusions. These inclusions are typically but not exclusively found in those brain regions that are predominantly affected. They are not limited to those neurones that are most likely to degenerate and can also be found in non-neuronal tissue. ${ }^{61}$ Furthermore, nuclear and cytosolic aggregates of huntingtin, the protein product of the Huntington's disease gene IT15, can also be found in nonneuronal tissue. This indicates that these aggregates are neither specific nor sufficient for cell death. Very recent evidence actually suggests that inclusion body formation reduces the levels of mutant huntingtin and the risk of neuronal cell death. Thus inclusion body formation could be interpreted as a "coping response" of the cell to toxic mutant huntingtin. ${ }^{62}$

Oligomerisation of expanded polyglutamine is not only a critical step in the formation of these inclusions, but it also stimulates different important cell death mechanisms previously identified in Huntington's disease, such as apoptosis and disturbed energy metabolism. Proapoptotic enzymes such as caspase 1 or 8 are activated in Huntington's disease and required for polyglutamine toxicity in cell culture models. Caspases can be inhibited pharmacologically by minocycline, and an influence of minocycline treatment on disease progression in Huntington mice was initially reported but subsequently not confirmed by others. ${ }^{63}{ }^{64}$ Lactate levels are raised and mitochondrial respiratory chain function is impaired in Huntington brain tissue, whereas creatine administration—which increases phosphocreatine levels and normalises mitochondrial function-leads to increased survival and delays motor symptoms in Huntington mice. ${ }^{65}$ Inhibition of the oligomerisation of expanded huntingtin by Congo red prevents ATP depletion and caspase activation, 
preserves normal cellular protein synthesis and degradation functions in vitro, and promotes the clearance of expanded huntingtin in vivo. ${ }^{66}$

Mutant huntingtin is more resistant to proteolysis, and aggregation of abnormal huntingtin is thus further promoted by insufficient breakdown of this protein by the proteasome pathway. ${ }^{67}$ Impaired function of the ubiquitin proteasome system cannot only be observed in brain tissue, but also in skin fibroblasts of Huntington's disease patients. ${ }^{68}$ Altered proteasomal function is also associated with disrupted mitochondrial membrane potential, released cytochrome c from mitochondria into the cytosol, and caspase activation. ${ }^{69}$ Normal function of the proteasome is closely linked to the machinery of molecular chaperones which mediate proper folding of other proteins and facilitate the transfer of misfolded proteins to the proteasome for degradation. Overexpression of chaperones such as Hsp 70 and Hsp 40 suppresses the aggregation and toxicity of polyglutamine containing proteins. For example, overexpression of Hsp70 in a mouse model of spinocerebellar ataxia l (SCAl) not only reduced pathological changes but also ameliorated the phenotype. ${ }^{67}$

A further "toxic gain of function" of mutant huntingtin is its interaction with transcriptional factors such as Spl and its coactivator TAFII 130 which in turn bind to a whole variety of genes such as neurotransmitter receptors and intracellular signalling systems. Coexpression of Spl and TAFIII30 in cultured striatal cells from wild type and Huntington transgenic mice reverses the transcriptional inhibition of the dopamine D2 receptor gene caused by mutant huntingtin and protects neurones from huntingtin induced cellular toxicity. ${ }^{62}$ Huntingtin, as well as other polyglutamine containing proteins, can also interact directly with other transcription factors such as the CREB binding protein (CBP), rendering them inactive. ${ }^{61} \mathrm{CBP}$ is one of several histone acetylases sequestered by polyglutamine inclusions. Histone acetylases are important gene expression regulators, and acetylation leads to increased mRNA transcription. CBP regulates the nuclear responses to a variety of cell signalling cascades including the neuronal response to neurotrophins, and overexpression of CBP rescues cells from polyglutamine toxicity. The incorporation of CBP into nuclear inclusions and resulting inactivation might therefore lead to a reduced capacity of the cells to respond to trophic factors essential for their survival. The incorporation and subsequent inactivation of other histone acetylases in the Huntington inclusions will further disturb the complicated gene expression network vital for the normal function and survival on neuronal cells. ${ }^{70} 71$ Histone acetylation itself can not currently be promoted pharmacologically but inhibitors of the physiological antagonist histone deacetylase markedly reduce polyglutamine induced toxicity. ${ }^{72}$

Loss of physiological function of huntingtin might also contribute to the pathogenesis of Huntington's disease, and increasing expression of wild type huntingtin in transgenic mice protects against the toxic effects of mutant huntingtin. Wild type huntingtin also shows antiapoptotic properties, possibly because wild type but not mutant huntingtin interacts with the proapoptotic protein HIPl. ${ }^{72}$ Wild type huntingtin also increases vesicular transport of brain derived neurotrophic factor (BDNF) along microtubules, but BDNF transport is impaired in the presence of mutant huntingtin or if the levels of wild type huntingtin are reduced.

\section{CONCLUSIONS}

Molecular genetic studies of familial Parkinson's disease have identified misfolding of proteins and failure of the proteasome to degrade such proteins as key events in the pathogenesis of Parkinson's disease. Significant challenges remain-namely, to extrapolate these findings to encompass the possible role of these identified genes and their gene products in the more common sporadic forms of the disease. Increasing knowledge and understanding of the identified genes and pathways are already being used to develop novel strategies for the treatment of this disease. Astounding progress has also been made in our understanding of the underlying mechanisms leading to cell death in Huntington's disease. A European-wide network, EURO-HD, has now been established which will facilitate drug trials aiming to identify disease modifying compounds.

\section{Authors' affiliations}

S M Hague, S Klaffke, O Bandmann, Academic Neurology Unit, Division of Genomic Medicine, University of Sheffield, UK

Competing interests: none declared

\section{REFERENCES}

1 Schrag A, Ben-Shlomo Y, Quinn NP. Cross sectional prevalence survey of idiopathic Parkinson's disease and Parkinsonism in London. BMJ 2000;321:21-22.

2 Polymeropoulos MH, Lavedan C, Leroy E, et al. Mutation in the alphasynuclein gene identified in families with Parkinson's disease. Science 1997;276:2045-7.

3 Kruger R, Kuhn W, Muller T, et al. Ala30Pro mutation in the gene encoding alpha-synuclein in Parkinson's disease. Nat Genet 1998;18:106-8.

4 Zarranz JJ, Alegre J, Gomez-Esteban JC, et al. The new mutation, E46K, of alpha-synuclein causes Parkinson and Lewy body dementia. Ann Neurol 2004:55:164-73.

5 Kobayashi H, Kruger R, Markopoulou K, et al. Haploinsufficiency at the alpha-synuclein gene underlies phenotypic severity in familial Parkinson's disease. Brain 2003; 126:32-42.

6 Golbe LI, Di lorio G, Sanges G, et al. Clinical genetic analysis of Parkinson's disease in the Contursi kindred. Ann Neurol 1996;40:767-75.

7 Spira PJ, Sharpe DM, Halliday G, et al. Clinical and pathological features of a Parkinsonian syndrome in a family with an Ala53Thr alpha-synuclein mutation. Ann Neurol 2001;49:313-19.

8 Kruger R, Kuhn W, Leenders KL, et al. Familial parkinsonism with synuclein pathology: clinical and PET studies of A3OP mutation carriers. Neurology 2000;56:1355-62.

9 Kruger R, Vieira-Saecker AM, Kuhn W, et al. Increased susceptibility to sporadic Parkinson's disease by a certain combined alpha-synuclein/ apolipoprotein E genotype. Ann Neurol 1999;45:611-17.

10 Khan N, Graham E, Dixon P, et al. Parkinson's disease is not associated with the combined alpha-synuclein/apolipoprotein E susceptibility genotype. Ann Neurol 2001:49:665-8.

11 Farrer M, Maraganore DM, Lockhart $P$, et al. Alpha-synuclein gene haplotypes are associated with Parkinson's disease. Hum Mol Genet 2001;10:1847-51.

12 Alves da Costa C. Recent advances on alpha-synuclein cell biology: functions and dysfunctions. Curr Mol Med 2003;3:17-24.

13 Papp MI, Kahn JE, Lantos PL. Glial cytoplasmic inclusions in the CNS of patients with multiple system atrophy (striatonigral degeneration, olivopontocerebellar atrophy and Shy-Drager syndrome). J Neurol Sci 1989;94:79-100

14 Abeliovich A, Schmitz Y, Farinas I, et al. Mice lacking alpha-synuclein display functional deficits in the nigrostriatal dopamine system. Neuron 2000;25:239-52.

15 Lansbury PT, Brice A. Genetics of Parkinson's disease and biochemical studies of implicated gene products. Curr Opin Cell Biol 2002;14:653-60.

16 Kirik D, Annett LE, Burger C, et al. Nigrostriatal alpha-synucleinopathy induced by viral vector-mediated overexpression of human alpha-synuclein: a new primate model of Parkinson's disease. Proc Natl Acad Sci USA 2003;100:2884-9.

17 Daver W, Kholodilov N, Vila M, et al. Resistance of alpha -synuclein null mice to the parkinsonian neurotoxin MPTP. Proc Natl Acad Sci USA 2002;99:14524-9.

18 Souza JM, Giasson BI, Chen Q, et al. Dityrosine cross-linking promotes formation of stable alpha-synuclein polymers. Implication of nitrative and oxidative stress in the pathogenesis of neurodegenerative synucleinopathies. J Biol Chem 2000;275:18344-9.

19 Xu J, Kao SY, Lee FJ, et al. Dopamine-dependent neurotoxicity of alphasynuclein: a mechanism for selective neurodegeneration in Parkinson disease. Nat Med 2002;8:600-6.

20 Singleton $\mathbf{A B}$, Farrer $M$, Johnson J, et al. Alpha-synuclein locus triplication causes Parkinson's disease. Science 2003;302:841.

21 Lucking CB, Durr A, Bonifati V, et al. Association between early-onset Parkinson's disease and mutations in the parkin gene. French Parkinson's Disease Genetics Study Group. N Engl J Med 2000;342:1560-7.

22 Klein C, Pramstaller PP, Kis B, et al. Parkin deletions in a family with adultonset, tremor-dominant parkinsonism: expanding the phenotype. Ann Neurol 2000:48:65-71. 
23 Khan NL, Brooks DJ, Pavese N, et al. Progression of nigrostriatal dysfunction in a parkin kindred: an [18F]dopa PET and clinical study. Brain 2002; 125:2248-56

24 Kuroda Y, Mitsui T, Akaike M, et al. Homozygous deletion mutation of the parkin gene in patients with atypical parkinsonism. J Neurol Neurosurg Psychiatry 2001;71:231-4.

25 West AB, Maraganore D, Crook J, et al. Functional association of the parkin gene promoter with idiopathic Parkinson's disease. Hum Mol Genet 2002;11:2787-92.

26 Leroy E, Boyer R, Auburger G, et al. The ubiquitin pathway in Parkinson's disease. Nature 1998;395:451-2.

27 Maraganore DM, Farrer MJ, Hardy JA, et al. Case-control study of the ubiquitin carboxy-terminal hydrolase L1 gene in Parkinson's disease. Neurology 1999:53:1858-60.

28 Ciechanover A, Orian A, Schwartz AL. The ubiquitin-mediated proteolytic pathway: mode of action and clinical implications. J Cell Biochem Suppl 2000;34:40-51.

29 Dawson TM, Dawson VL. Rare genetic mutations shed light on the pathogenesis of Parkinson disease. J Clin Invest 2003;111:145-51.

30 Stefanis L, Larsen KE, Rideout HJ, et al. Expression of A53T mutant but not wild-type alpha-synuclein in $\mathrm{PC} 12$ cells induces alterations of the ubiquitindependent degradation system, loss of dopamine release, and autophagic cell death. J Neurosci 2001;21:9549-60.

31 McNaught KS, Belizaire $\mathrm{R}$, Isacson $\mathrm{O}$, et al. Altered proteasomal function in sporadic Parkinson's disease. Exp Neurol 2003;179:38-46.

32 Hague S, Rogaeva E, Hernandez D, et al. Early-onset Parkinson's disease caused by a compound heterozygous DJ-1 mutation. Ann Neurol 2003;54:271-4.

33 Bonifati V, Breedveld GJ, Squitieri F, et al. Localization of autosomal recessive early-onset parkinsonism to chromosome 1p36 (PARK7) in an independent dataset. Ann Neurol 2002;51:253-6.

34 Miller DW, Ahmad R, Hague S, et al. L166P mutant DJ-1, causative for recessive Parkinson's disease, is degraded through the ubiquitin-proteasome system. J Biol Chem 2003;278:36588-95.

35 Yokota T, Sugawara K, Ito K, et al. Down regulation of DJ-1 enhances cell death by oxidative stress, ER stress, and proteasome inhibition. Biochem Biophys Res Commun 2003;312:1342-8.

36 Rizzu P, Hinkle DA, Zhukareva V, et al. DJ-1 colocalizes with tau inclusions: a link between parkinsonism and dementia. Ann Neurol 2004;55:113-18.

37 Neumann M, Muller V, Gorner K, et al. Pathological properties of the Parkinson's disease-associated protein DJ-1 in alpha-synucleinopathies and tauopathies: relevance for multiple system atrophy and Pick's disease. Acto Neuropathol (Berl) 2004;107:489-96.

38 Valente EM, Abou-Sleiman PM, Caputo V, et al. Hereditary early-onset Parkinson's disease caused by mutations in PINK1. Science 2004;304:1158-60.

39 Rohe CF, Montagna P, Breedveld G, et al. Homozygous PINK1 C-terminus mutation causing early-onset parkinsonism. Ann Neurol 2004;56:427-31.

40 Maraganore DM, Wilkes K, Lesnick TH, et al. A limited role for DJ 1 in Parkinson disease susceptibility. Neurology 2004;63:550-3.

41 Healy DG, Abou-Sleiman PM, Ahmadi KR, et al. The gene responsible for PARK6 Parkinson's disease, PINK1, does not influence common forms of parkinsonism. Ann Neurol 2004;56:329-35.

42 Paisan-Ruiz C, Jain S, Evans EW, et al. Cloning of the Gene Containing Mutations that Cause PARK8-Linked Parkinson's Disease. Neuron 2004;44:595-600.

43 Zimprich A, Biskup S, Leitner P, et al. Mutations in LRRK2 Cause Autosomal Dominant Parkinsonism with Pleomorphic Pathology. Neuron 2004;44:601-7.

44 Nichols WC, Pankratz N, Hernandez D, et al. Genetic screening for a single common LRRK2 mutation in familial Parkinson's disease. Lancet 2005:365:410-12

45 Di Fonzo A, Rohe CF, Ferreira J, et al. A frequent LRRK2 gene mutation associated with autosomal dominant Parkinson's disease. Lancet 2005;365:412-15.

46 Gilks WP, Abou-Sleiman PM, Gandhi S, et al. A common LRRK2 mutation in idiopathic Parkinson's disease. Lancet 2005;365:415-16.

47 Le WD, Xu P, Jankovic J, et al. Mutations in NR4A2 associated with familial Parkinson disease. Nat Genet 2003;33:85-9.
48 McCormack AL, Thiruchelvam M, Manning-Bog AB, et al. Environmental risk factors and Parkinson's disease: selective degeneration of nigral dopaminergic neurons caused by the herbicide paraquat. Neurobiol Dis 2002;10:119-27.

49 Betarbet R, Sherer TB, MacKenzie G, et al. Chronic systemic pesticide exposure reproduces features of Parkinson's disease. Nat Neurosci 2000;3:1301-6.

50 Hoglinger GU, Feger J, Prigent A, et al. Chronic systemic complex I inhibition induces a hypokinetic multisystem degeneration in rats. J Neurochem 2003;84:491-502

51 Gao HM, Hong JS, Zhang W, et al. Synergistic dopaminergic neurotoxicity of the pesticide rotenone and inflammogen lipopolysaccharide: relevance to the etiology of Parkinson's disease. J Neurosci 2003;23:1228-36.

52 Orr CF, Rowe DB, Halliday GM. An inflammatory review of Parkinson's disease. Prog Neurobiol 2002;68:325-40.

53 Teismann P, Tieu K, Choi DK, et al. Cyclooxygenase-2 is instrumental in Parkinson's disease neurodegeneration. Proc Natl Acad Sci USA 2003;100:5473-8

54 McNaught KS, Pearl DP, Brownell AL, et al. Systemic exposure to proteasome inhibitors causes a progressive model of Parkinson's disease. Ann Neurol 2004;56: 149-62.

55 McLean PJ, Kawamata H, Hyman BT. $\alpha$-synuclein-enhanced green fluorescent protein fusion proteins form proteasome sensitive inclusions in primary neurons. Neuroscience 2001;104:901-12.

56 Auluck PK, Meulener MC, Bonini NM. Mechanisms of Suppression of alpha synuclein Neurotoxicity by Geldanamycin in Drosophila. J Biol Chem 2005;280:2873-8.

57 Farrer $M$, Maraganore DM, Lockhart $P$, et al. alpha-Synuclein gene haplotypes are associated with Parkinson's disease. Hum Mol Genet 2001;10:1847-51

58 Yamada M, Mizuno Y, Mochizuki H. Parkin gene therapy for alphasynucleinopathy: a rat model of Parkinson's disease. Hum Gene Ther 2005; 16:262-70.

59 Temussi PA, Masino L, Pastore A. From Alzheimer to Huntington: why is a structural understanding so difficult? Embo J 2003;22:355-61

60 Squitieri F, Gellera C, Cannella M, et al. Homozygosity for CAG mutation in Huntington disease is associated with a more severe clinical course. Brain 2003; 126:946-55.

61 Ross CA, Poirier MA, Wanker EE, et al. Polyglutamine fibrillogenesis: the pathway unfolds. Proc Natl Acad Sci USA 2003;100:1-3.

62 Arrasate M, Mitra S, Schweitzer ES, et al. Inclusion body formation reduces levels of mutant huntingtin and the risk of neuronal death. Nature 2004;431:805-10.

63 Hughes RE, Olson JM. Therapeutic opportunities in polyglutamine disease. Nat Med 2001;7:419-23.

64 Smith DL, Woodman B, Mahal A, et al. Minocycline and doxycycline are not beneficial in a model of Huntington's disease. Ann Neurol 2003:54:186-96.

65 Andreassen OA, Dedeoglu A, Ferrante RJ, et al. Creatine increase survival and delays motor symptoms in a transgenic animal model of Huntington's disease. Neurobiol Dis 2001;8:479-91.

66 Sanchez I, Mahlke C, Yuan J. Pivotal role of oligomerization in expanded polyglutamine neurodegenerative disorders. Nature 2003:421:373-9.

67 Muchowski PJ. Protein misfolding, amyloid formation, and neurodegeneration: a critical role for molecular chaperones? Neuron 2002;35:9-12

$68 \mathrm{Seo} \mathrm{H}$, Sonntag KC, Isacson O. Generalized brain and skin proteasome inhibition in Huntington's disease. Ann Neurol 2004:56:319-28.

69 Jana NR, Zemskov EA, Wang G, et al. Altered proteasomal function due to the expression of polyglutamine-expanded truncated $\mathrm{N}$-terminal huntingtin induces apoptosis by caspase activation through mitochondrial cytochrome c release. Hum Mol Genet 2001;10:1049-59.

70 Dunah AW, Jeong H, Griffin A, et al. Spl and TAFII130 transcriptional activity disrupted in early Huntington's disease. Science 2002;296:2238-43.

71 Hughes RE. Polyglutamine disease: acetyltransferases awry. Curr Biol 2002;12:R141-3.

72 Cattaneo E, Rigamonti D, Goffredo D, et al. Loss of normal huntingtin function: new developments in Huntington's disease research. Trends Neurosci $2001 ; 24: 182-8$. 